\title{
Simultaneous TMS-fMRI of the Visual Cortex Reveals Functional Network, Even in Absence of Phosphene Sensation
}

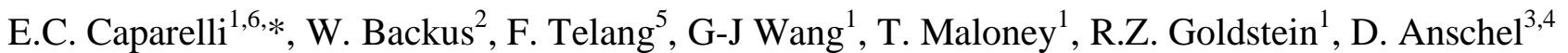 \\ and F. Henn ${ }^{1}$
}

\author{
${ }^{l}$ Medical Department, Brookhaven National Laboratory, Upton, NY, USA \\ ${ }^{2}$ Department of Anesthesiology, Stony Brook University, Stony Brook, NY, USA \\ ${ }^{3}$ St. Charles Hospital, Port Jefferson, NY, USA \\ ${ }^{4}$ American Neuromonitoring Associates, Columbia, MD, USA \\ ${ }^{5}$ National Institute on Alcohol Abuse and Alcoholism, Bethesda, MD, USA \\ ${ }^{6}$ SCAN Center, Stony Brook University, Stony Brook, NY, USA
}

\begin{abstract}
Phosphene sensation is commonly used to measure cortical excitability during transcranial magnetic stimulation (TMS) of the occipital cortex. However, some individuals lack this perception, and the reason for it is still unknown. In this work, we used functional magnetic resonance imaging (fMRI) to detect brain activation during local TMS of the occipital cortex in twelve healthy subjects. We found that TMS modulated brain activity in areas connected to the stimulation site, even in people unable to see phosphene. However, we observed a trend for a lower bloodoxygenation-level dependent (BOLD) signal, and smaller brain-activation clusters near the stimulated site than in the interconnected brain areas, suggesting that TMS pulse is more effective downstream than at its application site. Furthermore, we noted prominent differences in brain activation/deactivation patterns between subjects who perceived phosphene and those who did not, implying a functional distinction in their neuronal networks that might explain the origin of differences in phosphene generation.
\end{abstract}

Keywords: BOLD, fMRI, TMS, phosphene.

\section{INTRODUCTION}

Transcranial magnetic stimulation (TMS), a non-invasive method, is used to explore the human brain's processes, such as language, vision, and motor systems [1]. Applying TMS pulses to the occipital cortex entails the brief perception of a light flash, called phosphene sensation [2], a response that is employed extensively to assess the excitability of the primary visual cortex [3]. However, the origin of phosphene generation is unknown, and its induction with single-pulse TMS is irregular [4-7]. Apparently, phosphenes are generated in V1, the primary visual cortex, and V2/3, the extrastriate visual-cortical areas $[2,5,6]$, indicating that the primary visual cortex is one fundamental area for phosphene perception [7,8]; nevertheless, others studies point to its subcortical origin (e.g., optic radiation) [9]. Meister and colleagues [10] found remarkable differences in fMRIactivation during visual stimulation (checkerboard paradigm) between people perceiving phosphene and those lacking this perception, suggesting that this disparity might reflect interindividual functional differences in visual neuronal networks. To verify this supposition, we interleaved TMS pulses in occipital cortices and 4-Tesla whole-brain blood oxygenation

*Address correspondence to this author at the Brookhaven National Laboratory, Medical Dept., Bldg. 490, Upton, NY 11973, USA;

Tel: (631) 344-3640; Fax: (631) 344-7671; E-mail: caparelli@bnl.gov level dependent (BOLD)-fMRI acquisition to image local and distant co-activations, and reveal their association with phosphene generation.

Concurrent TMS-fMRI, a non-invasive approach demonstrating the transsynaptical propagation of activation between and area stimulated by a TMS pulse, and its remote interconnected brain regions, reveals direct causal functional interactions between these two sites in the brain [11,12]. Accordingly, this dual modality can highlight the brain's adaptation to the TMS-induced virtual lesion and the consequent changes in activity associated with the behavioural changes frequently observed throughout TMS cortical stimulation [13]. Measurements of such changes in tissue oxygen, neural activity, and total hemoglobin (electrophysiology, optical imaging), in the visual cortex of cats, demonstrated that the TMS stimulus modifies neuronal activity that is reflected in alterations in cerebral hemodynamics and, therefore, can be detected by fMRI [14]. Some groups explored the effects of the TMS pulse on brain function after applying the TMS-fMRI combination to the human motor cortex [15-26]. Similarly, this combination was applied to the prefrontal cortices [27], frontal eye-field (FEF) [28], and parietal cortices [29,30]. However, to our knowledge, no one employed fMRI to evaluate the brain's response when discharging TMS directly over the occipital area. In this work, we used simultaneous TMS-fMRI to map 
the functional response in the cortical- and subcortical-areas, associated with phosphene generation, after delivering the TMS pulse over the primary visual cortex.

\section{METHODS}

\section{Subjects}

Twelve non-smoking adult healthy volunteers (11 men; 1 woman; age $32 \pm 5$ years) participated in this study. All subjects were carefully screened to ensure that they met the study's inclusion criteria; viz., age 18 - 40 years, ability to read and speak English fluently, and right-handed dominance. Subjects were excluded if they were taking any medication other than vitamins (or contraceptives), had any history of chronic medical-/neuropsychiatric-illnesses or drug use/ dependence, had any contra-indicated metallic objects in the body, claustrophobia, or positive urine toxicology on the day of the study. The Institution Review Board (IRB) at Stony Brook University approved the study protocol; each participant signed the IRB's approved informed consent form.

\section{TMS-Setup}

Before the TMS-fMRI scan, we administered a singlepulse TMS, using the Magstim, Model 220 (The Magstim Company, Wales, UK - pulse duration $250 \mu \mathrm{s}$, maximum repetition rate $1 \mathrm{~Hz}$, maximum field strength $2.0 \mathrm{~T}$ ), with a standard $70 \mathrm{~mm}$ double coil $(70-\mathrm{mm}$ outer wing diametermaximum field strength $2.2 \mathrm{~T}$ ). The stimulus was given over the left occipital area (primary visual cortex); the participants, keeping their eyes open, wore a blindfold to minimize external visual input. Varying the intensity of the stimulus in steps of 5\%, we asked participants to report whenever they saw phosphene; we defined a subject's phosphene threshold as the level at which it was elicited in three of five trials. This procedure took place in the subject-preparation room, in the MRI building (local magnetic field $0.6 \mathrm{mT}$ ).

\section{TMS-fMRI Session}

In the RF-shielded MRI room, TMS was applied through a nonferromagnetic double coil (70-mm outer wing diametermaximum field strength $0.9 \mathrm{~T}$ ) connected to the same magnetic stimulator used during the setup session (above), via a 10 meter cable through a radio-frequency (RF) filter tube. The TMS coil was attached inside the RF head coil. Each subject lay supine with their head resting on the MRIcompatible TMS coil (the center of which was positioned at the occipital bone's most prominent projection, slightly lateral to the left side of the midline) inside the RF-coil (local magnetic field $1 \mathrm{~T}$ ). We verified each volunteers' threshold for phosphene sensation (with their eyes open but covered). Next, the volunteer was moved into the MRI scanner (local magnetic field $4 \mathrm{~T}$ ) and their phosphene threshold rechecked. During simultaneous TMS-fMRI, again the TMS stimulus was applied over the left occipital region with the intensity set for the phosphene threshold under $4 \mathrm{~T}$; for subjects unable to perceive phosphene, we set the stimulator at a fixed value of $40 \%$, i.e., the phosphene threshold measured for the first subject able to perceive phosphene under $4 \mathrm{~T}$.

The stimulus paradigm comprised three 30 -second epochs of "REST", and three with "TMS" (28-seconds long; 8 TMS pulses); during the latter, the pulse was applied with a frequency of $0.25 \mathrm{~Hz}$ interleaved, after $300 \mathrm{~ms}$, with fMRI acquisition [31]. Subjects pushed a button, with their right hand, every time they saw "flashes of light". TMS pulses and fMRI data acquisition were synchronized using a TTL (5 V) trigger signal generated immediately after acquiring the previous image in the time series.

\section{MRI}

Subjects underwent fMRI in a 4 Tesla Varian/Siemens MRI scanner, equipped with a self-shielded whole-body SONATA gradient set and a standard quadrature head resonator. Functional images were collected using a singleshot $\mathrm{T} 2 *$-weighted gradient-recalled-echo planar imaging (GRE-EPI) imaging sequence (TE/TR=20/2000 ms, $4 \mathrm{~mm}$ slice thickness, $1 \mathrm{~mm}$ gap, typically 33 coronal slices, $64 \times 64$ matrix size, $3.125 \mathrm{~mm}$ in-plane resolution, $90^{\circ}$-flip angle, 94 time points) covering the whole brain. Within $2 \mathrm{~s}$ of TR, the image was acquired in 1.7 s, i.e., $0.3 \mathrm{~s}$ after the previous image acquisition. Padding minimized the volunteers' head motion during the scans.

\section{fMRI Data Processing}

We analyzed the fMRI data sets on UNIX and LINUX workstations (Compaq Alpha and dual XEON), using interactive data language (IDL) (Research Systems, Boulder, $\mathrm{CO}$ ) and statistical parametric mapping packages, version two (SPM2 - Wellcome Department of Cognitive Neurology, London).

Primary images of the raw data were reconstructed using IDL. EPI's reconstruction program uses phase correction to deghost the time series [32], an iterative phase-correction to minimize signal loss [33], and a Hamming filter to improve the signal-to-noise ratio [34]. The first four sequential time points of each time series was eliminated from further analyses to ensure a steady state of magnetization. All time series were motion-corrected to the first volume using 6 degrees of freedom (3 rotations, 3 translations). A B-splineinterpolation was used for re-slicing the motion-corrected data sets. For Talairach normalization, the individual scans were matched to a standard reference brain of the same contrast, using a voxel size of $3 \times 3 \times 3 \mathrm{~mm}^{3}$. All time series were spatially smoothed with an $8 \mathrm{~mm}$ Gaussian Kernel.

\section{Statistical Analyses}

The activation maps were calculated for the entire group using a generalized linear model (GLM - fixed-effect analysis). The design matrix was generated by a boxcar reference function, and its derivative convolved with the canonic hemodynamic response function (HRF). The time series were band-pass-filtered with a high-pass filter (cut-off frequencies: $1 / 128 \mathrm{~Hz}$ ), and an HRF as low-pass filter. The mean signal-intensity was not scaled to avoid false deactivation signals [35,36]. Clusters with at least 15 voxels and $p<0.05$ (FWE) were considered significant in the group analyses [37].

\section{RESULTS}

\section{Behavioural Data}

We found a significant change in the phosphene threshold under different background static magnetic field for the six subjects who perceived phosphene. Fig. (1) shows 


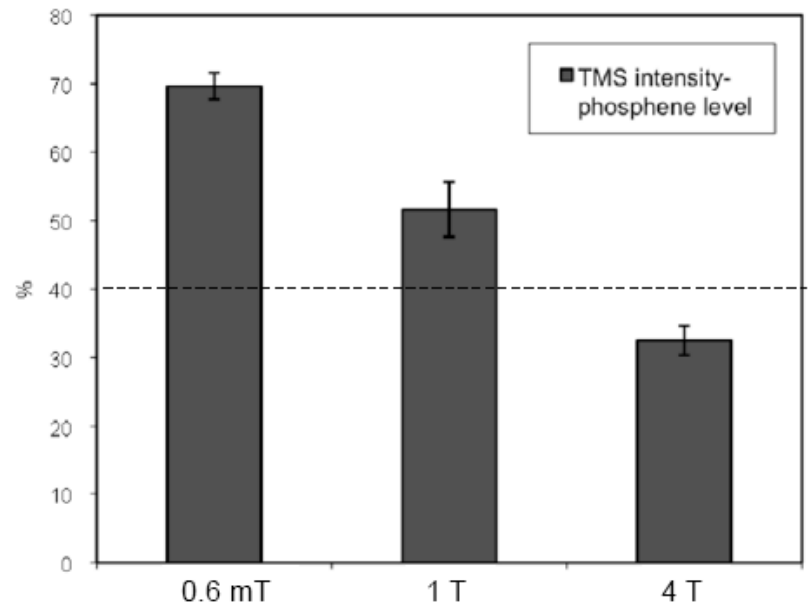

Fig. (1). Average of phosphene level, for six subjects, for three different static magnetic-fields: $0.6 \mathrm{mT}$ (subject-preparation room), $1 \mathrm{~T}$ (MRI scanner bed outside the MRI), and $4 \mathrm{~T}$ (center of the MRI scanner). The dashed line, at $40 \%$, shows the TMS intensity chosen for the TMS stimulus during TMS-fMRI acquisition for group 2. The error bars denote the standard errors.

the average of the phosphene threshold for three such conditions: $0.6 \mathrm{mT}$ (subject-preparation room), $1 \mathrm{~T}$ (MRI scanner bed outside the MRI), and $4 \mathrm{~T}$ (center of the MRI scanner). For these six volunteers, the phosphene threshold decreased under an increasing static magnetic field.

The performance data, i.e., button-press frequency, during the fMRI-TMS session shows that the subjects experienced the phosphene sensation for about $50 \%$ of the TMS pulses applied (average: $48.6 \%$, standard deviation: $27.1 \%$ ).

\section{Brain Activation}

Fig. (2A and B), illustrates the effects of the TMS stimulus in brain activation (positive BOLD signal). The fixed-effects analysis revealed significant brain activation for group 1 (able to see phosphenes - Fig. 2A, Table 1) at the following locations: Near the stimulus site (left occipital, cuneus - $[-3$, -90, 15]), occipital (precuneus), parietal (precuneus and subgyral), frontal lobe areas (precentral gyrus - frontal eyes-field (FEF) [38], middle-, medial-, and superior-frontal gyrus, sub-gyral), cerebellum (cerebellar tonsil), brainstem (midbrain), thalamus, insula, cingulate gyrus, and uvula. Conversely, significant brain activation for the second group (non-perceiving phosphenes - Fig. 2B, Table 1) occurred in the subcortical occipital area (cuneus - $[-24,-72,9])$, parietal lobe (sub-gyral), temporal lobe (middle and superior temporal gyrus), frontal lobe areas (precentral, middle-, medial-, and superior-frontal gyrus, sub-gyral), cerebellum (pyramis and inferior semi-lunar lobule), thalamus, and insula.

We also evaluated the negative BOLD signal (deactivation). In those perceiving phosphene, there was significant deactivation in the occipital (middle occipital gyrus, temporal lobe (middle temporal gyrus and sub-gyral), frontal lobe (precentral gyrus, middle- and superior-frontal gyrus, sub-gyral), cerebellum (posterior lobe, inferior semilunar lobule), and limbic lobe (anterior cingulate) (Fig. 2A, Table 2). For those lacking phosphene perception, Fig. 2B, Table $\mathbf{2}$ lists the significantly deactivated areas in the occipital lobe (fusiform gyrus, middle occipital gyrus), frontal lobe areas (middle-, medial-, and superior-frontal gyrus, sub-gyral), cerebellum (declive), and the limbic lobe (parahippocampal gyrus, anterior- and posterior-cingulate).

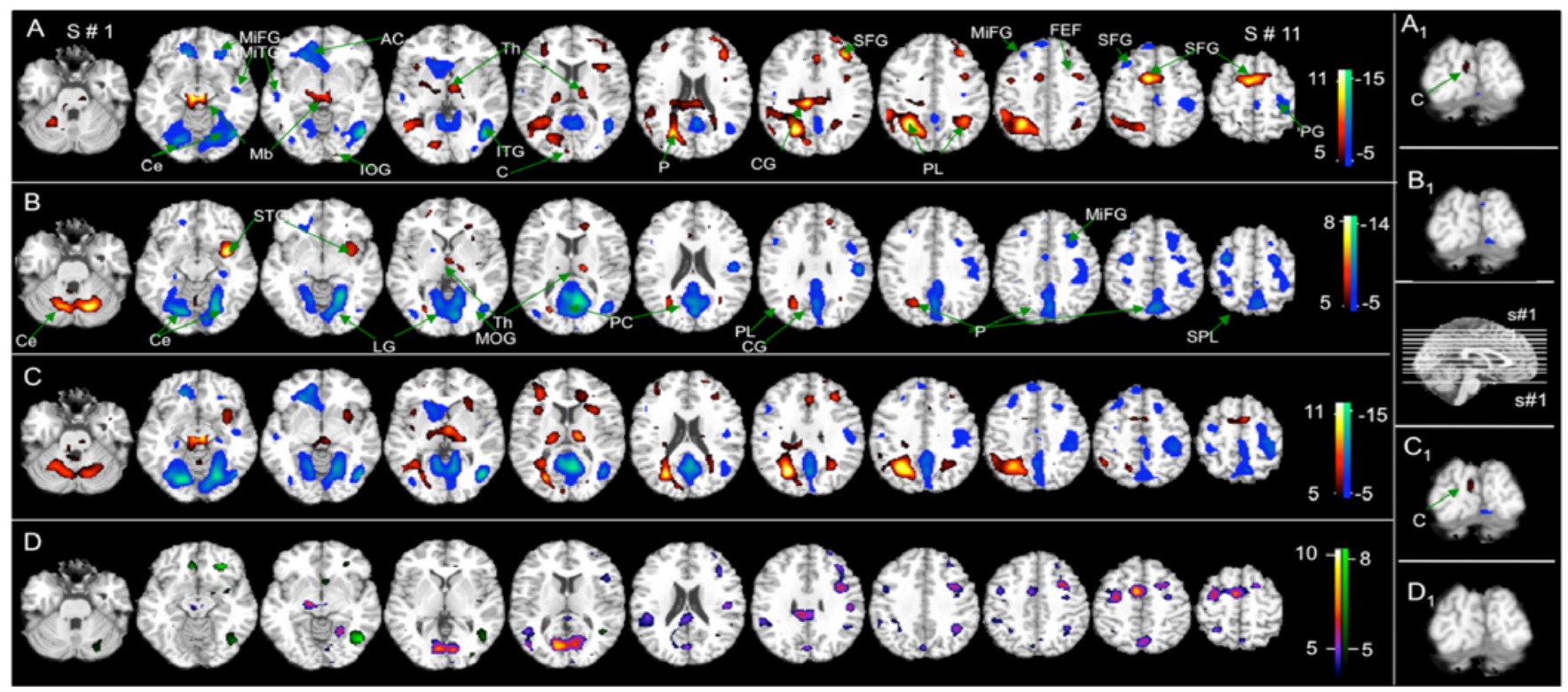

Fig. (2). Statistical maps of BOLD signal resulting from a fix-effect analysis for the two groups, 1) those able to see phosphenes (Fig. A), and, 2) those unable to see phosphenes (Fig. B) (6 subjects in each group). Fig. C shows the conjunctive analysis: $\mathrm{C}=\mathrm{Phosphene}(\mathrm{P})+$ nophosphene (NP) and D the comparison between groups: D = P > NP (purple), NP > P (green). The color bar shows the T-score window; FWE, $p<0.05$, activation (red - yellow) and deactivation (blue - green). FEF $=$ Frontal Eyes-Field, Mb $=$ midbrain, $\mathrm{STG}=\mathrm{superior}$ temporal gyrus, TL=Temporal Lobe, AC=anterior cingulated, LG=lingual Gyrus, Th=Thalamus, ITG=Inferior Temporal Gyrus, MOG = Middle Occiptal Gyrus, IOG=Inferior Occiptal Gyrus, PC=Posterior Cingulate, P=Precunious, CG=Cingulate Gyrus, MiTG=Middle Temporal Gyrus, SFG=Superior Frontal Gyrus, PG=Precentral Gyrus, MiFG=Middle Frontal Gyrus, PL = Parietal Lobe, SPL=Superior Parietal Lobe, $\mathrm{C}=$ Cuneus. Coronal views showing the activation near the stimulated site for groups $1\left(\mathrm{~A}_{1}\right), 2\left(\mathrm{~B}_{1}\right)$, conjunctive analysis $\left(\mathrm{C}_{1}-\right.$ highlighted in grey in Table 3 ) and differential analysis $\left(\mathrm{D}_{1}\right)$; a sagittal view with the position of the axial slices presented in Figs. A, B, C, and $\mathbf{D}$. 
Table 1. Brain Regions Showing Activation for Group 1 and 2 (Fig. 2A, B); Results from a Fix-Effects Analysis, T $\geq 4.56$ (FWE, $p<$ 0.05), Voxel Size $\geq 15$. $(*[38])$

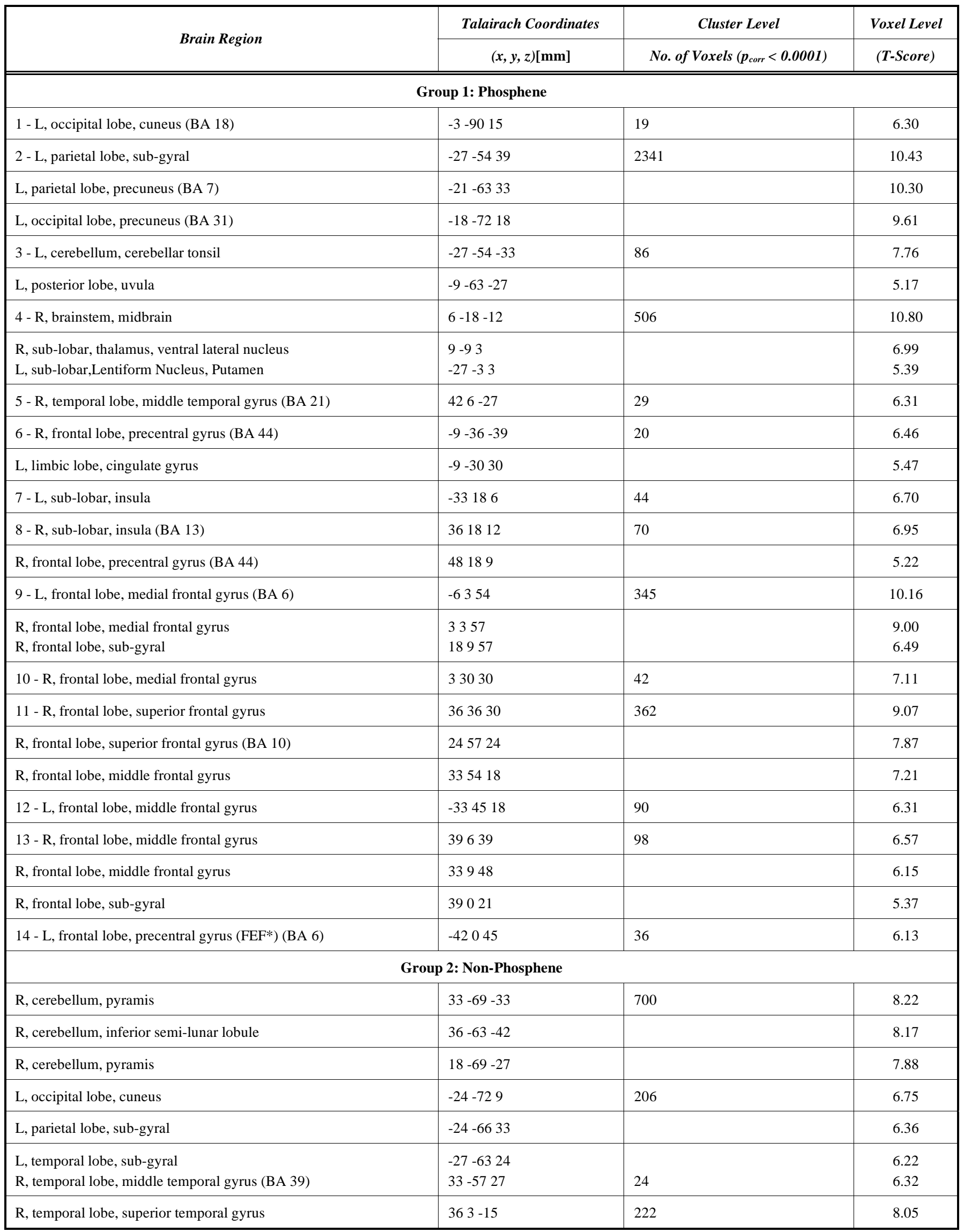


Table 1. contd.....

\begin{tabular}{|c|c|c|c|}
\hline \multirow{2}{*}{ Brain Region } & Talairach Coordinates & Cluster Level & Voxel Level \\
\hline & $(x, y, z)[m m]$ & No. of Voxels $($ pcorr $<0.0001)$ & (T-Score) \\
\hline R, sub-lobar, insula (BA 13) & 33210 & & 5.05 \\
\hline $\begin{array}{l}\mathrm{R} \text {, sub-lobar, thalamus, lateral posterior nucleus } \\
\mathrm{R} \text {, sub-lobar, thalamus }\end{array}$ & $\begin{array}{l}15-1812 \\
3-93\end{array}$ & 74 & $\begin{array}{l}6.37 \\
6.23\end{array}$ \\
\hline $\begin{array}{l}\mathrm{L} \text {, sub-lobar, insula (BA 13) } \\
\mathrm{L} \text {, sub-lobar, insula } \\
\mathrm{L} \text {, frontal lobe, precentral gyrus (BA 44) } \\
\mathrm{R} \text {, frontal lobe, sub-gyral } \\
\mathrm{R} \text {, frontal lobe, middle frontal gyrus }\end{array}$ & $\begin{array}{l}-42-36 \\
-36-126 \\
-4299 \\
24366 \\
3039-6\end{array}$ & $\begin{array}{l}38 \\
66\end{array}$ & $\begin{array}{l}5.60 \\
5.19 \\
5.27 \\
5.92 \\
4.85\end{array}$ \\
\hline L, frontal lobe, middle frontal gyrus (BA 10) & -395418 & 39 & 5.96 \\
\hline $\begin{array}{l}\mathrm{L} \text {, frontal lobe, middle frontal gyrus } \\
\mathrm{L} \text {, frontal lobe, middle frontal gyrus (BA 9) } \\
\mathrm{R} \text {, frontal lobe, superior frontal gyrus } \\
\mathrm{R} \text {, frontal lobe, superior frontal gyrus } \\
\mathrm{R} \text {, frontal lobe, medial frontal gyrus (BA 9) }\end{array}$ & $\begin{array}{l}-424230 \\
-423636 \\
-21543 \\
184230 \\
213624\end{array}$ & $\begin{array}{l}30 \\
26\end{array}$ & $\begin{array}{l}5.29 \\
5.06 \\
6.17 \\
5.74 \\
4.89\end{array}$ \\
\hline
\end{tabular}

Table 2. Brain Regions Showing Deactivation for Group 1 and 2 (Fig. 2A, B); Results from a Fix-Effects Analysis, T $\geq 4.56$ (FWE, $p$ $<0.05$ ), Voxel Size $\geq 15$

\begin{tabular}{|c|c|c|c|}
\hline \multirow{2}{*}{ Brain Region } & Talairach Coordinates & Cluster Level & Voxel Level \\
\hline & $(x, y, z)[\mathrm{mm}]$ & No. of Voxels $\left(p_{\text {corr }}<0.0001\right)$ & (T-Score) \\
\hline \multicolumn{4}{|c|}{ Group 1: Phosphene } \\
\hline $\mathrm{R}$, occipital lobe, middle occipital gyrus & $48-66-3$ & 1961 & 15.24 \\
\hline $\mathrm{L}$, cerebellum, posterior lobe, declive & $-21-78-15$ & & 12.58 \\
\hline $\begin{array}{l}\mathrm{R} \text {, cerebellum, posterior lobe, declive } \\
\mathrm{L} \text {, cerebellum, inferior semi-lunar lobule }\end{array}$ & $\begin{array}{l}21-78-15 \\
-3-57-48\end{array}$ & 16 & $\begin{array}{c}10.99 \\
5.16\end{array}$ \\
\hline $\begin{array}{l}\mathrm{L} \text {, temporal lobe, middle temporal gyrus } \\
\mathrm{R} \text {, temporal lobe, sub-gyral } \\
\mathrm{R} \text {, temporal lobe, sub-gyral }\end{array}$ & $\begin{array}{l}-54-18-3 \\
45-24-15 \\
45-9-12\end{array}$ & $\begin{array}{l}39 \\
24\end{array}$ & $\begin{array}{l}6.84 \\
5.93 \\
5.41\end{array}$ \\
\hline $\mathrm{L}$, limbic lobe, anterior cingulate & $-933-12$ & 487 & 11.26 \\
\hline $\mathrm{L}$, frontal lobe, middle frontal gyrus & $-2136-9$ & & 10.62 \\
\hline $\mathrm{L}$, frontal lobe, sub-gyral & $-1827-6$ & & 9.62 \\
\hline $\mathrm{R}$, frontal lobe, middle frontal gyrus (BA 11) & $3033-12$ & 40 & 9.14 \\
\hline $\begin{array}{l}\mathrm{R}, \text { frontal lobe, precentral gyrus (BA 4) } \\
\mathrm{R}, \text { frontal lobe, precentral gyrus (BA 4) } \\
\mathrm{R}, \text { frontal lobe, precentral gyrus (BA 4) }\end{array}$ & 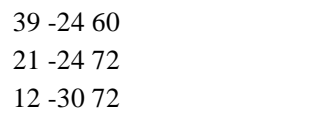 & 507 & $\begin{array}{l}8.10 \\
7.64 \\
7.14\end{array}$ \\
\hline L, frontal lobe, superior frontal gyrus (BA 8) & -64848 & 79 & 8.38 \\
\hline L, frontal lobe, superior frontal gyrus (BA 8) & -362151 & 81 & 8.07 \\
\hline L, frontal lobe, superior frontal gyrus (BA 8) & -273348 & & 6.48 \\
\hline \multicolumn{4}{|c|}{ Group 2: Non-Phosphene } \\
\hline $\mathrm{R}$, limbic lobe, posterior cingulate & $9-6012$ & 5043 & 14.01 \\
\hline $\mathrm{R}$, occipital lobe, fusiform gyrus & $21-60-9$ & & 12.79 \\
\hline $\mathrm{R}$, cerebellum, declive & $24-69-12$ & & 12.56 \\
\hline
\end{tabular}


Table 2. contd.....

\begin{tabular}{|c|c|c|c|}
\hline Brain Region & Talairach Coordinates & Cluster Level & Voxel Level \\
\hline L, occipital lobe, middle occipital gyrus (BA 19) & $-39-786$ & 80 & 8.09 \\
\hline $\mathrm{L}$, frontal lobe, middle frontal gyrus & -481827 & 25 & 6.23 \\
\hline $\mathrm{L}$, frontal lobe, middle frontal gyrus & $-1839-12$ & 61 & 7.34 \\
\hline $\begin{array}{l}\text { L, frontal lobe, sub-gyral } \\
\text { L, limbic lobe, anterior cingulated (BA 32) }\end{array}$ & $\begin{array}{ll}-18 & 21-3 \\
-12 & 45-6\end{array}$ & & $\begin{array}{l}5.42 \\
5.17\end{array}$ \\
\hline $\mathrm{R}$, limbic lobe, parahippocampal gyrus & $33-27-15$ & 38 & 7.07 \\
\hline
\end{tabular}

Conjunctive analysis (Fig. 2C and Table 3) showed significant activation for all participants at the occipital (cuneus), parietal (precuneus), temporal (middle temporal and superior frontal gyrus, sub-gyral), frontal lobe (middle-, medial-, and superior-frontal gyrus), brainstem (midbrain), thalamus, insula, and cingulate gyrus. Conspicuous deactivation was evident in the occipital, frontal lobe areas (middle-, and superior-frontal gyrus), cerebellum, temporal lobe and limbic lobe (posterior and anterior cingulate, parahippocampal gyrus), and also in the occipital, frontal lobe areas (middle-, and superior-frontal gyrus), cerebellum, temporal lobe and limbic lobe (posterior- and anteriorcingulate, parahippocampal gyrus). Furthermore, the differential BOLD signal (Fig. 2D and Table 4) revealed that activation is more significant for the phosphene-perceiving group than those who do not see it (group $1>$ group 2). This was evident in the occipital (cuneus, lingual gyrus, middle occipital gyrus), parietal (paracentral superior- and inferiorlobules, supramarginal- and postcentral-gyrus), temporal (middle temporal gyrus), frontal lobe areas (paracentral- and precentral-lobules, middle-, medial-, superior-frontal gyrus), brainstem (midbrain), cerebellum (culmen), and the limbic lobe (parahippocampal gyrus). In contrast, for the nonphosphene-perceiving group (group $2>$ group 1) activation was more pronounced in the occipital (middle occipital gyrus), temporal (middle temporal gyrus), frontal lobe areas (middle- and medial-frontal gyrus), cerebellum (tuber and culmen), and the limbic lobe (amygdala).

\section{DISCUSSION}

In this study we have used the simultaneous TMS-fMRI combination to evaluate the functional response associated with phosphene perception after delivering a TMS stimulus over the brain's primary visual cortex area. We found that TMS modulated brain activity even in absence of phosphene generation; however, the remarkable difference in brain activation/deactivation between the people who perceive phosphene and those who lack this perception (Fig. 2) implies substantial divergence in their function networks; such divergence was already suggested in a previous study [10], and may explain the difference in phosphene generation.
TMS activated a smaller network in group 2 than in group 1, with no activation at the stimulus site; however, some brain areas were activated in both groups, including the subcortical occipital and parietal regions (Fig. 2, Table 1). In addition, BOLD change was less significant (Fig. 2) near the stimulus site than in distant brain regions, suggesting that TMS effects are more effective in interconnected brain areas than at the stimulation site. Early work, reviewed by Siebner and colleagues [39], pointed out that while single pulses or short trains of TMS at intensities below motor threshold failed to induce changes in the BOLD signal at the site of stimulation, the signal increased in the stimulated primary motor cortex (M1) during supra-threshold TMS. Therefore, as they suggested, the latter observation may reflect activation due to afferent feedback from TMS-induced hand movements. In a similar context, we noted the presence of BOLD signal near the site of stimulation only for those subjects who perceive phosphene, suggesting that this activation might trigger a feedback response from their visualizing phosphenes.

The results from group 1 reveal a direct connection between the FEF, the occipital area, and the parietal cortex (Fig. 2, Table 1). The link between the first two was documented by a positron emission tomography (PET) study in primates, viz., delivering TMS in FEF causes changes in cerebral blood flow (CBF) in the visual cortex [40]. Similarly, Ruff and colleagues demonstrated a connection between FEF and the occipital cortex [28], and later between parietal cortex and the occipital cortex [29], by stimulating the FEF and the parietal cortex respectively. However, in both cases, their acquisition of fMRI by MRI surface coils at the occipital cortex greatly reduced BOLD-fMRI sensitivity in its more anterior regions (e.g., parietal and frontal cortex), virtually confining the effect to the occipital area (V1-V4). A different stimulation technique, transcranial electric stimulation (TES), also activated the occipital-, parietal-, and prefrontal- (FEF and supplementary eyes-field (SEF)) cortices, and areas associated with pain or discomfort, such as the limbic lobe, insula, and thalamus, when the electrodes were positioned on the occipital area [41]. Although TES preferentially stimulates fibers rather than cell bodies, this 
Table 3. Brain Regions Showing Activation and Deactivation for the Conjunctive Analysis $(P+N P, P=P h o s p h e n e$ and NP $=$ NonPhosphene) (Fig. 2C); Results from a Fix-Effects Analysis, T $\geq 4.56$ (FWE, $p<0.05$ ), voxel size $\geq 15$. (* $p_{\text {corr }}$ not Statistically Significant)

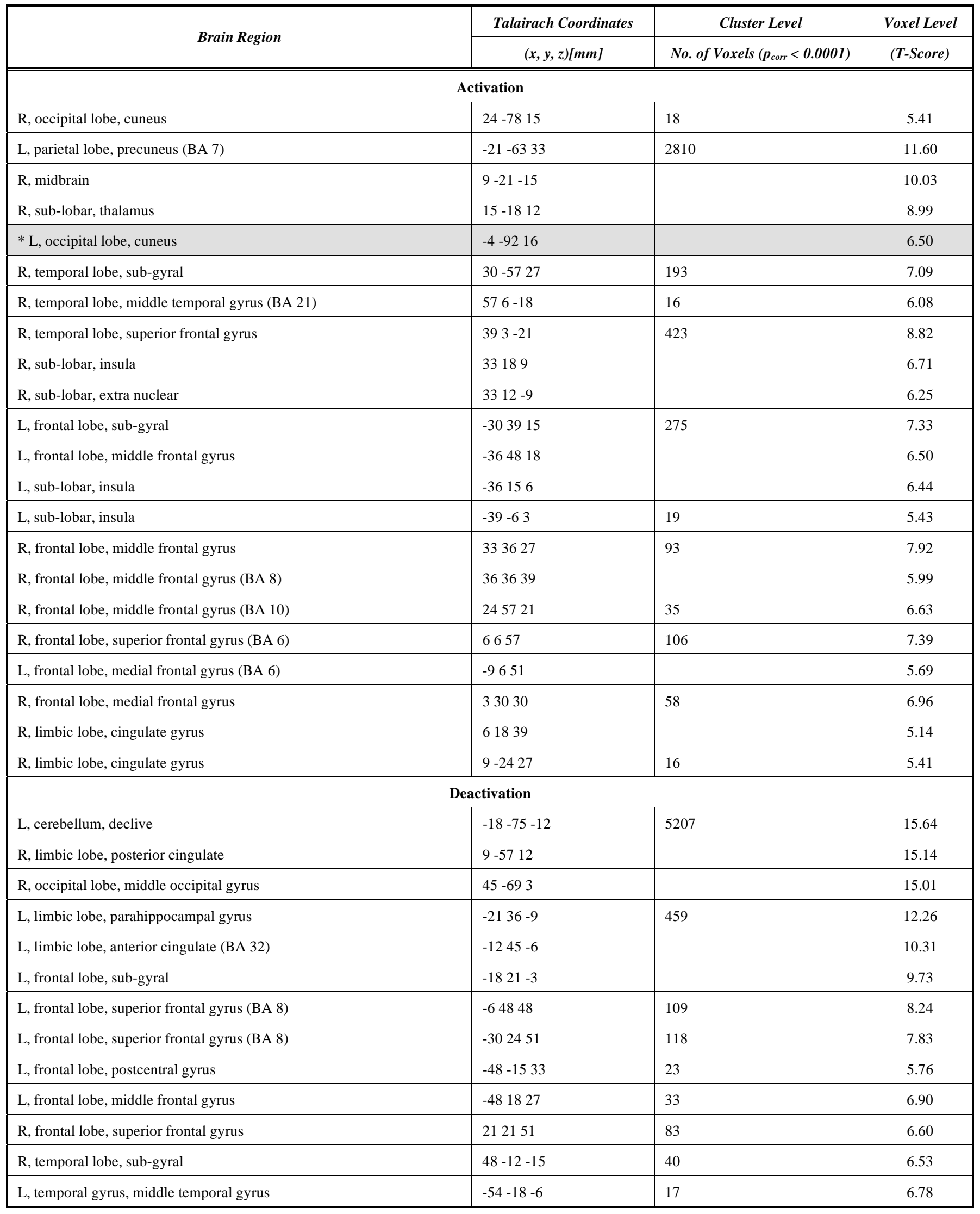


Table 4. Brain Regions Showing the Differential Results for $P>$ NP, and NP > P (Fig. 2D); Results from a Fix-Effects Analysis, T $\geq$ 4.56 (FWE, $p<0.05$ ), Voxel Size $\geq 15$

\begin{tabular}{|c|c|c|c|}
\hline \multirow{2}{*}{ Brain Region } & Talairach Coordinates & Cluster Level & Voxel Level \\
\hline & $(x, y, z)[\mathrm{mm}]$ & No. of Voxels $\left(p_{\text {corr }}<0.0001\right)$ & (T-Score) \\
\hline \multicolumn{4}{|c|}{$\mathbf{P}>\mathbf{N P}$} \\
\hline L, occipital lobe, cuneus (BA 18) & $-12-7215$ & 949 & 10.60 \\
\hline $\mathrm{L}$, occipital lobe, lingual gyrus & $-6-783$ & & 8.59 \\
\hline R, occipital lobe, cuneus (BA 18) & $12-6915$ & & 8.43 \\
\hline $\mathrm{R}$, occipital lobe, lingual gyrus & $15-96-9$ & 18 & 5.64 \\
\hline $\mathrm{R}$, occipital lobe, cuneus & $9-966$ & & 5.63 \\
\hline L, occipital lobe, middle occipital gyrus & $-45-759$ & 370 & 6.98 \\
\hline $\mathrm{L}$, temporal lobe, middle temporal gyrus & $-45-549$ & & 6.28 \\
\hline $\mathrm{L}$, parietal lobe, inferior parietal lobule & $-51-3927$ & & 6.26 \\
\hline $\mathrm{L}$, brainstem, midbrain & $-12-15-6$ & 85 & 8.28 \\
\hline $\mathrm{R}$, brainstem, midbrain & $3-18-9$ & & 6.39 \\
\hline R, limbic lobe, parahippocampal gyrus (BA 19) & $24-54-6$ & 45 & 7.43 \\
\hline $\mathrm{R}$, limbic lobe, parahippocampal gyrus & $33-27-15$ & 32 & 5.89 \\
\hline $\mathrm{R}$, cerebellum, anterior lobe, culmen & $24-2724$ & & 5.83 \\
\hline $\mathrm{L}$, frontal lobe, medial frontal gyrus & -6054 & 999 & 9.55 \\
\hline $\mathrm{R}$, frontal lobe, superior frontal gyrus & 21957 & & 8.60 \\
\hline $\mathrm{L}$, frontal lobe, middle frontal gyrus & -30654 & & 8.30 \\
\hline $\mathrm{R}$, frontal lobe, precentral gyrus (BA 44) & 48189 & 31 & 5.82 \\
\hline $\mathrm{L}$, frontal lobe, paracentral lobule (BA 6) & $-6-2775$ & 76 & 5.85 \\
\hline L, frontal lobe, precentral gyrus (BA 4) & $-24-2472$ & & 5.84 \\
\hline L, parietal lobe, paracentral lobule (BA 6) & $-3-3369$ & & 5.71 \\
\hline $\mathrm{L}$, parietal lobe, superior parietal lobule & $-27-5163$ & 31 & 6.64 \\
\hline $\mathrm{R}$, parietal lobe, inferior parietal lobule (BA 40) & $39-4839$ & 102 & 7.05 \\
\hline R, parietal lobe, supramarginal gyrus (BA 40) & $54-4527$ & & 5.81 \\
\hline $\mathrm{R}$, parietal lobe, postcentral gyrus & $48-1824$ & 57 & 7.30 \\
\hline \multicolumn{4}{|c|}{$\mathbf{N P}>\mathbf{P}$} \\
\hline $\mathrm{R}$, occipital lobe, middle occipital gyrus & $36-876$ & 22 & 6.94 \\
\hline $\mathrm{R}$, occipital lobe, middle occipital gyrus & $48-63-6$ & 200 & 8.63 \\
\hline $\mathrm{R}$, temporal lobe, middle temporal gyrus & $45-549$ & & 6.35 \\
\hline $\mathrm{R}$, temporal lobe, middle temporal gyrus & $39-6021$ & & 5.02 \\
\hline $\mathrm{R}$, cerebellum, posterior lobe, tuber & $27-75-30$ & 72 & 6.15 \\
\hline $\mathrm{L}$, cerebellum, anterior lobe, culmen & $-12-51-18$ & 16 & 5.37 \\
\hline $\mathrm{R}$, limbic lobe, parahippocampal gyrus, amygdala & $210-15$ & 22 & 5.83 \\
\hline $\mathrm{R}$, sub-lobar, extra nuclear & $615-6$ & 19 & 5.60 \\
\hline $\mathrm{R}$, frontal lobe, middle frontal gyrus & $3036-12$ & 47 & 8.30 \\
\hline $\mathrm{L}$, frontal lobe, medial frontal gyrus & $-636-12$ & 34 & 7.01 \\
\hline
\end{tabular}




\begin{tabular}{|c|c|c|c|}
\hline \multirow{2}{*}{ Brain Region } & Talairach Coordinates & Cluster Level & Voxel Level \\
\hline & $(x, y, z)[\mathrm{mm}]$ & No. of Voxels $($ pcorr $<0.0001)$ & (T-Score) \\
\hline L, frontal lobe, middle frontal gyrus (BA 9) & -423636 & 23 & 5.45 \\
\hline $\mathrm{L}$, frontal lobe, middle frontal gyrus (BA 46) & -454218 & & 5.18 \\
\hline $\mathrm{L}$, frontal lobe, middle frontal gyrus (BA 9) & -483330 & & 5.10 \\
\hline
\end{tabular}

earlier work supports our findings on the interconnection among the occipital area, parietal cortices, and the FEF. However, brain activation in the thalamus, insula, and cingulate gyrus in both of our groups, i.e., areas previously associated with discomfort by those authors [41], suggest that the BOLD response therein is not caused by the TMS stimulus ; rather, it may be a consequence of any unpleasant sensation, such as the "tap" that participants felt during stimulation.

Brainstem activation, in the midbrain area, was significant only in the phosphene-perceiving group (Fig. 2, Tables 1 and 4). This part of the midbrain has a conduit function; for example, the superior colliculus is directly associated with visual reflexes, and receives input from the eyes [42]. The red nucleus, another of its structures, controls motor coordination [43]. Therefore, the activation of the midbrain only in the phosphene group (Fig. 2, Table 1) conforms to their perceiving phosphene and their subsequent button-pressing, which, we believe, also was responsible for activating the motor area (L, medial frontal gyrus (BA 6)).

The cerebellum was activated in both groups, but significantly more so in group 2 (Fig. 2, Table 1). Researchers believed that the cerebellum is devoted exclusively to motor functions [44]; recently, many other roles were proposed, including its serving as an internal timing system, assessing millisecond intervals [45]. Our results support the latter suggestion because we noted high activation in the cerebellum of the non-phosphene group that never undertakes any motor task during the scan; possibly this group is more attentive to the interval between the TMS pulse and image acquisition than the other group who might focus more on the phosphene sensation.

Non-phosphene perceivers (group 2) exhibited larger deactivated brain areas than perceivers (group 1) (Fig. 2, Table 2). Previous fMRI studies suggested concomitant deactivation might represent the active suppression of neural activity in brain regions that are unimportant or may interfere with performing the required tasks [46-48]. In contrast, other work hypothesizes that fMRI deactivation may simply reflect a direct hemodynamic change in the vascular system in response to variation in the adjacent regional cerebral blood flow ( $\mathrm{rCBF}),[49]$. Additionally, our previous results suggest that changes in $\mathrm{rCBF}$ are taskdependent [48]. Furthermore, previous concurrent repetitive TMS (rTMS) and PET studies demonstrated that rTMS can induce decrease or increase of $\mathrm{rCBF}$, depending on the parameters of the stimulus $[50,51]$. Therefore, the negative BOLD response we observed in group 1 may reflect local changes in $\mathrm{rCBF}$ induced by the TMS stimulus because the activated/deactivated areas appear to be balanced (Fig. 2, Table 1 and 2). However, for groups 2, neither of these two hypotheses explain the extended deactivated network; rather, they suggest a TMS-induced overall reduction in brain activity.

The decline in the phosphene threshold with an increase in the static magnetic field (Fig. 1) may indicate a change in the performance of the TMS coil, or in cortical excitability under a high static magnetic field. Testing the coil's performance with a pickup coil, in the same position relative to the TMS coil, inside and outside the MRI scanner, revealed no marked change in induced voltage, (data not shown), pointing to the second possibility. However, the small sample and the inaccuracy of the method of registering the phosphene threshold (self-reported) preclude a definitive statement. A longitudinal study encompassing more volunteers is needed to validate this preliminary finding; more precise results would emerge from motor-evoked potential measurements, obtained by applying TMS over the motor area, under different static magnetic fields. Another limitation of our study was associated with the behavioural differences between group during the experiment; while the perceiver group was actively engaged in a task (selfmonitored, pressing a button on seeing phosphene), the nonperceiver group was not. Apart from likely differences in attention between the groups (that may well explain the BOLD differences in posterior brain regions), the perceiver group also performed an action (button press). These behavioural differences might have also contributed to the observed differences in the results, rather than uniquely affected by the difference in phosphene perception. Therefore, future studies where subjects indicate whether or not they saw phosphene, during TMS stimulus, are suggested.

\section{CONCLUSION}

Our work demonstrated that TMS modulates brain activity in interconnected areas in people who see phosphene and even in those who lack this perception. Brain activation in areas interconnected with the stimulated site seemingly present a higher T-score with larger cluster sizes (Fig. 2) than those near the site of stimulation, indicating that the effect of the TMS stimulus intensifies in these remote areas. Therefore, a sub-threshold stimulus will not greatly affect the stimulated area, but can significantly do so in remote brain regions. Moreover, the general variation in the patterns of brain activation/deactivation between people who perceive phosphene and those who lack this perception points to a possible difference in their neuronal network that may explain the cause of the difference in phosphene generation. 


\section{ACKNOWLEDGEMENTS}

We thank Dr. D. Tomasi for the helpful comments. This work was supported by the Laboratory Directed Research and Development Program of Brookhaven National Laboratory (LDRD-BNL), National Institutes of Health (GCRC MO1RR-10710-06S1) and U. S. Department of Energy (DOE OBER).

\section{NOTE}

This manuscript has been authored by Brookhaven Science Associates, LLC under Contract No. DE-AC0298CHI-886 with the U.S. Department of Energy. The United States Government retains, and the publisher, by accepting the article for publication, acknowledges, a world-wide license to publish or reproduce the published form of this manuscript, or allow others to do so, for the United States Government purposes.

\section{REFERENCES}

[1] Terao Y, Ugawa Y. Basic mechanisms of TMS. J Clin Neurophysiol 2002; 19: 322-43.

[2] Kammer T. Phosphenes and transient scotomas induced by magnetic stimulation of the occipital lobe: their topographic relationship. Neuropsychologia 1999; 37: 191-8.

[3] Machii K, Cohen D, Ramos-Estebanez C, et al. Safety of rTMS to non-motor cortical areas in healthy participants and patients. Clin Neurophysiol 2006; 117: 455-71.

[4] Kammer T, Puls K, Erb M, et al. Transcranial magnetic stimulation in the visual system. II. Characterization of induced phosphenes and scotomas. Exp Brain Res 2005; 160: 129-40.

[5] Kammer T, Beck S, Erb M, et al. The influence of current direction on phosphene thresholds evoked by transcranial magnetic stimulation. Clin Neurophysiol 2001; 112: 2015-21.

[6] Kastner S, Demmer I, Ziemann U. Transient visual field defects induced by transcranial magnetic stimulation over human occipital pole. Exp Brain Res 1998; 118: 19-26.

[7] Cowey A, Walsh V. Magnetically induced phosphenes in sighted, blind and blindsighted observers. Neuroreport 2000; 11: 3269-73.

[8] Pascual-Leone A, Walsh V. Fast backprojections from the motion to the primary visual area necessary for visual awareness. Science 2001; 292: 510-12.

[9] Epstein CM, Verson R, Zangaladze A. Magnetic coil suppression of visual perception at an extracalcarine site. J Clin Neurophysiol 1996; 13: 247-52.

[10] Meister IG, Weidemann J, Dambeck N, et al. Neural correlates of phosphene perception. Suppl Clin Neurophysiol 2003; 56: 305-11.

[11] Sack AT, Linden DE. Combining transcranial magnetic stimulation and functional imaging in cognitive brain research: possibilities and limitations. Brain Res Brain Res Rev 2003; 43: 41-56.

[12] Caparelli EC. TMS\&fMRI - A new neuroimaging combinational tool to study brain function. CMIR 2007; 3: 109-15.

[13] Bestmann S, Ruff CC, Blankenburg F, et al. Mapping causal interregional influences with concurrent TMS-fMRI. Exp Brain Res 2008; 191: 383-402.

[14] Allen E, Pasley BN, Duong T, et al. Transcranial magnetic stimulation elicits coupled neural and hemodynamic consequences. Science 2007; 317: 1918-21.

[15] Bohning DE, Shastri A, McConnell KA, et al. A combined TMS/fMRI study of intensity-dependent TMS over motor cortex. Biol Psychiatry 1999; 45: 385-94.

[16] Bohning DE, Shastri A, Nahas Z, et al. Echoplanar BOLD fMRI of brain activation induced by concurrent transcranial magnetic stimulation. Invest Radiol 1998; 33: 336-40.

[17] Bohning DE, Shastri A, Wassermann EM, et al. BOLD-f MRI response to single-pulse transcranial magnetic stimulation (TMS). J Magn Reson Imaging 2000; 11: 569-574.

[18] Bohning DE, Shastri A, McGavin L, et al. Motor cortex brain activity induced by $1-\mathrm{Hz}$ transcranial magnetic stimulation is similar in location and level to that for volitional movement. Invest Radiol 2000; 35: 676-83.
[19] Bohning DE, Shastri A, Lomarev MP, et al. BOLD-fMRI response vs. transcranial magnetic stimulation (TMS) pulse-train length: testing for linearity. J Magn Reson Imaging 2003; 17: 279-90.

[20] Denslow S, Bohning DE, Bohning PA, et al. An increased precision comparison of TMS-induced motor cortex BOLD fMRI response for image-guided versus function-guided coil placement. Cogn Behav Neurol 2005; 18: 119-26.

[21] Denslow S, Lomarev M, George MS, et al. Cortical and subcortical brain effects of transcranial magnetic stimulation (TMS)-induced movement: an interleaved TMS/functional magnetic resonance imaging study. Biol Psychiatry 2005; 57: 752-60.

[22] Baudewig J, Siebner HR, Bestmann S, et al. Functional MRI of cortical activations induced by transcranial magnetic stimulation (TMS). Neuroreport 2001; 12: 3543-48

[23] Bestmann S, Baudewig J, Siebner HR, et al. Subthreshold highfrequency TMS of human primary motor cortex modulates interconnected frontal motor areas as detected by interleaved fMRITMS. Neuroimage 2003; 20: 1685-96.

[24] Bestmann S, Baudewig J, Siebner HR, et al. Functional MRI of the immediate impact of transcranial magnetic stimulation on cortical and subcortical motor circuits. Eur J Neurosci 2004; 19: 1950-62.

[25] Bestmann S, Oliviero A, Voss M, et al. Cortical correlates of TMSinduced phantom hand movements revealed with concurrent TMSfMRI. Neuropsychologia 2006; 44: 2959-71.

[26] Bestmann S, Baudewig J, Siebner HR, et al. BOLD MRI responses to repetitive TMS over human dorsal premotor cortex. Neuroimage 2005; 28: 22-9.

[27] Nahas Z, Lomarev M, Roberts DR, et al. Unilateral left prefrontal transcranial magnetic stimulation (TMS) produces intensitydependent bilateral effects as measured by interleaved BOLD fMRI. Biol Psychiatry 2001; 50: 712-20.

[28] Ruff CC, Blankenburg F, Bjoertomt O, et al. Concurrent TMSfMRI and psychophysics reveal frontal influences on human retinotopic visual cortex. Curr Biol 2006; 16: 1479-88.

[29] Ruff CC, Bestmann S, Blankenburg F, et al. Distinct causal influences of parietal versus frontal areas on human visual cortex: evidence from concurrent TMS fMRI. Cereb Cortex 2008; 18: 81727.

[30] Sack AT, Kohler A, Bestmann S, et al. Imaging the brain activity changes underlying impaired visuospatial judgments: simultaneous fMRI, TMS, and behavioral studies. Cereb Cortex 2007; 17: 284152.

[31] Caparelli EC, Backus W, Telang F, et al. The advances of combining transcranial magnetic stimulation and fMRI in a high field. In ISMRM Workshop on Advances in High Field MR. Pacific Grove, CA, USA 2007.

[32] Buonocore MH, Gao L. Ghost artifact reduction for echo plannar imaging using image phase correction. Magn Reson Med 1997; 38 : 89-100.

[33] Caparelli EC, Tomasi D. K-space spatial low-pass filters can increase signal loss artifacts in Echo-planar imaging. Biomed Signal Process Control 2008; 3: 107-14.

[34] Hamming RW. Digital filters. Englewood Cliffs, NJ: Prentice-Hall, Inc. 1982.

[35] Aguirre GK, Zarahn E, D'Esposito M. The inferential impact of global signal covariates in functional neuroimaging analyses. Neuroimage 1998; 8: 302-6.

[36] Gavrilescu M, Shaw ME, Stuart GW, et al. Simulation of the effects of global normalization procedures in functional MRI. Neuroimage 2002; 17: 532-42.

[37] Friston KJ, Worsley KJ, Frackowiak RSJ, et al. Assessing the significance of focal activations using their spatial extent. Hum Brain Mapp 1994; 1: 214-20.

[38] Paus T. Location and function of the human frontal eye-field: a selective review. Neuropsychologia 1996; 34: 475-83.

[39] Siebner HR, Lee L, Bestmann S. Interleaving TMS with functional MRI: now that it is technically feasible how should it be used? Clin Neurophysiol 2003; 114: 1997-99.

[40] Paus T, Jech R, Thompson CJ, et al. Transcranial magnetic stimulation during positron emission tomography: a new method for studying connectivity of the human cerebral cortex. J Neurosci 1997; 17: 3178-84.

[41] Brandt SA, Brocke J, Röricht S, et al. In vivo assessment of human visual system connectivity with transcranial electrical stimulation during functional magnetic resonance imaging. Neuroimage 2001; 14: 366-75. 
[42] Hoffmann KP. Conduction velocity in pathways from retina to superior colliculus in the cat: a correlation with receptive-field properties. J Neurophysiol 1973; 36: 3 .

[43] van Kan PL, McCurdy ML. Discharge of primate magnocellular red nucleus neurons during reaching to grasp in different spatial locations. Exp Brain Res 2002; 142: 151-7.

[44] Gilman S, Lechtenberg R, Bloedel JR. Disorders of the cerebellum. Philadelphia, PA, Ed. Davis, FA 1981.

[45] Koch G, Oliveri M, Torriero S, et al. Repetitive TMS of cerebellum interferes with millisecond time processing. Exp Brain Res 2007; 179: 291-9.

[46] Laurienti PJ, Burdette JH, Wallace MT, et al. Deactivation of sensory-specific cortex by cross-modal stimuli. J Cogn Neurosci 2002; 14: 420-29.

[47] Lewis JW, Beauchamp MS, DeYoe EA. A comparison of visual and auditory motion processing in human cerebral cortex. Cereb Cortex 2000; 10: 873-88.
[48] Tomasi D, Ernst T, Caparelli EC, et al. Common deactivation patterns during working memory and visual attention tasks: an intra-subject fMRI study at 4 Tesla. Hum Brain Mapp 2006; 27: 694-705.

[49] Shmuel A, Yacoub E, Pfeuffer J, et al. Sustained negative BOLD, blood flow and oxygen consumption response and its coupling to the positive response in the human brain. Neuron 2002; 36: 1195 210.

[50] Knoch D, Treyer V, Regard M, et al. Lateralized and frequencydependent effects of prefrontal rTMS on regional cerebral blood flow. Neuroimage 2006; 31: 641-8.

[51] Speer AM, Willis MW, Herscovitch P, et al. Intensity-dependent regional cerebral blood flow during $1-\mathrm{Hz}$ repetitive transcranial magnetic stimulation (rTMS) in healthy volunteers studied with H215O positron emission tomography: II. Effects of prefrontal cortex rTMS. Biol Psychiatry 2003; 54: 826-32.

(C) Caparelli et al.; Licensee Bentham Open.

This is an open access article licensed under the terms of the Creative Commons Attribution Non-Commercial License (http://creativecommons.org/licenses/by-nc/3.0/) which permits unrestricted, non-commercial use, distribution and reproduction in any medium, provided the work is properly cited. 